\title{
Interference Effect of Downstream Strip-Plate on the Crossflow Vibration of a Square Cylinder*
}

\author{
Yusuke KAWABATA**, Tsutomu TAKAHASHI***, \\ Takeshi HAGINOYA**** and Masataka SHIRAKASHI***** \\ ** Institute of Ocean Energy, Saga University, 1-Honjo, Saga, 840-8502, Japan \\ E-mail: kawabata@ioes.saga-u.ac.jp \\ ${ }^{* * *}$ Nagaoka University of Technology, Kamitomioka 1603-1, Nagaoka, Niigata, Japan. \\ **** NISSAN MOTOR CO., LTD., 560-2, Okatsukoku, Atsugi-shi, Kanagawa 243-0192, Japan \\ Malaysia-Japan International Institute of Technology, Universiti Teknologi Malaysia, Jalan Semarak, \\ 54100 Kuala Lumpur, Malaysia.
}

\begin{abstract}
Wind tunnel experiments are carried out to investigate the interference effect of a downstream strip-plate of width $w=d$ on the crossflow vibration of a square cylinder of side length $d=26 \mathrm{~mm}$. While the vibration induced by the Karman vortex is insufficiently suppressed by plates with crossflow height less than $l_{d}=4 d$, the galloping is suppressed even by a square plate, i.e. $l_{d}=d$, at gap ratios $1<s / d<$ 2.0. When a plate of $l_{d} \leq 2 d$ is located at a gap ratio $s / d<1-1.4$, a large vibration occurs at $U>5 \mathrm{~m} / \mathrm{s}$ with maximum amplitude around $s / d=0.3$. This amplitude increases divergently with increasing flow velocity. Measurement of the lift force on the square cylinder and the velocity in the near wake for the corresponding fixed system indicates that the vibration is not caused by periodic flow changes. The quasi-static hypothesis predicts that the vibration is caused by fluid-elastic instability but not by a periodic vortex shedding. Hence, the name 'Wake Body Interference Fluid Elastic Vibration (WBIFEV)' is proposed for this vibration.
\end{abstract}

Key words: Flow-Induced Vibration, Fluid-Elastic Vibration, Interference Effect, Cruciform Arrangement

\section{Introduction}

Square cross-sectional cylinders (square cylinders) in uniform flow are known to vibrate in the crossflow direction. This vibration is induced by the Karman vortex (hence its name, Karman vortex-induced vibration, abbreviated to KVIV) and by fluid-elastic instability called galloping. Numerous studies have been conducted on suppressing these vibrations and various techniques such as placing another cylinder downstream in tandem arrangement ${ }^{(1-5)}$, attaching small bodies to the vibrating cylinder ${ }^{(4,6)}$ and small 2-D aerofoil-shaped fairings at both up- and down-stream ends of the vibrating body ${ }^{(6)}$ are known to be effective. On the other hand, tandem placement of an identical counterpart can enhance the vibration ${ }^{(5)}$. Most of these methods are aimed at two-dimensional flow control. Recently, it was reported that vibrations can be suppressed or created by the three-dimensional interference effect of a downstream cylinder or a strip-plate in a cruciform arrangement. In this setup, nothing is attached to the vibrating cylinder and the flow is interfered by a body downstream of the cylinder.

Bae et al. ${ }^{(7)}$ reported that KVIV of a circular cylinder is suppressed by setting an identical counterpart downstream in cruciform arrangement. They also identified another vibration is induced at velocities above the KVIV range by longitudinal vortices periodically shedding around the crossing (LVIV: longitudinal vortex-induced vibration).

*Received 27 Aug., 2013 (No. T2-10-0673) Japanese Original : Trans. Jpn. Soc. Mech. Eng., Vol.77, No.775, B (2011), pp.647-658 (Received 20 Aug., 2010) [DOI: 10.1299/jfst.8.348]

Copyright (C) 2013 by JSME 
Later, two types of longitudinal vortices were recognised; trailing vortex (TV) and necklace vortex (NV) with different configurations and shedding frequencies. These vortex types are interchangeable by varying the gap-to-diameter ratio $s / d$, where $d$ is the diameter of the upstream cylinder and $s$ is the gap between the two cylinders ${ }^{(8)}$.

Koide et al. ${ }^{(9)}$ replaced the downstream cruciform arrangement circular cylinder with a strip-plate of width $w=0.5 d-1.75 d$, expecting that the plate would offer better flow control, and investigated its influence on the vibration of the upstream circular cylinder. In a series of wind tunnel experiments, they found that the strip-plate exerts greater KVIV suppression than the circular cylinder; specifically, the effect of a strip-plate of $w / d=0.5$ is comparable to that of a circular cylinder of diameter $d$. They also reported that KVIV is enhanced by downstream strip-plates of width $w \leq d$ over a certain range of $s / d$. The range of $s / d$ being dependent on $w / d$, both classes of LVIV can be induced by the strip-plate. If the strip-plate satisfies $w / d \geq 0.62$, vibrations are induced only by the TV (termed trailing vortex induced vibration; TVIV).

Koide et al. ${ }^{(10)}$ also investigated the interference effect of the cruciform downstream strip-plate on the crossflow vibrations, i.e. KVIV and galloping, of a square cylinder and reported as follows. KVIV and galloping are severely suppressed by very long strip-plates of width $w=0.5 d-d$ (where $d$ is the side length of the square cylinder) and KVIV can be enhanced similarly to a circular cylinder. LVIV is induced at around $s / d=1.4$, possibly by the trailing vortex.

The present authors ${ }^{(11)}$ investigated similarity of the above phenomena in systems of different dimensions. In the previous paper, the same phenomena were reported regardless of the system dimensions and increasing $w / d$ increased the range of $s / d$ that suppressed galloping. Visualization experiments of flows on the square cylinder surface showed that LVIV reported by Koide is induced by the trailing vortex.

The above research suggests that cylinder vibrations can be controlled by the three-dimensional interference effect of a downstream body. To date, experiments have adopted a downstream cylinder or strip-plate of infinite length, i.e. the downstream body spans the entire height of the measuring section of wind tunnel. From a practical viewpoint, the length of the downstream body must be known and a smaller length is desirable.

In the current work, a strip-plate of width $w=d$ and finite length $l_{d}$, as short as $l_{d}=d$, is placed downstream of a square cylinder. The aim was to investigate how the finite-length plate influences crossflow vibrations on the cylinder, with a view to developing a vibration control technique.

\section{Experimental apparatus and measurements}

Arrangement of the experimental apparatus and the coordinate system used in this study are shown in Fig.1. The square cross-section and length of the test section is $0.32 \mathrm{~m} \times 0.32 \mathrm{~m}$ and $1 \mathrm{~m}$, respectively (the upper and lower walls of the test section are not shown in Fig.1). A square cylinder of side length $d=26 \mathrm{~mm}$ and effective length $L=315 \mathrm{~mm}$ passes through slots on the side walls of the measuring section and is elastically supported by two twin-plate-springs at both ends outside the measuring section, so that it moves in a crossflow direction. The effect of flow through the slots is removed by end plates attached to the cylinder. A strip-plate of width $w=d$, thickness $t=5 \mathrm{~mm}$ and length $l_{d}$ is placed downstream of the square cylinder, fixed rigidly to an L-shaped support that is mounted on a traversing device below the measuring section enabling adjustment of the gap $s$. In our previous work $^{(11)}$, the strip-plate spanned the entire height of the measuring section, which is indicated as $l_{d} / d=\infty$ in the current paper.

The free flow velocity $U$ is measured by a ring-type velocimeter ${ }^{(12)}$. The $x$-component of the velocity $u$ is measured by a hot-wire probe placed at an appropriate location to detect 
the periodic vortex shedding $(x / d=1.5, y / d=1.25, z / d=0.4)$. The displacement $Z$ of the square cylinder is measured at each end by laser displacement sensors, which separately record the translational and rotational motions. The lift force on the fixed square cylinder $F_{L}$ is measured by a load cell. The natural frequency $f_{n}$ and the logarithmic damping factor $\delta$ are obtained from free damping oscillations in otherwise stationary air. Experimental conditions are listed in Table 1. The oscillation frequency $f_{z}$ is confirmed to be constant and coincides with $f_{n}$ irrespective of $s / d$ and $U$ throughout all experiments. The L-shaped support without the strip-plate, i.e. $w / d=l_{d} / d=0.38$ is confirmed to not affect KVIV and galloping of the square cylinder.

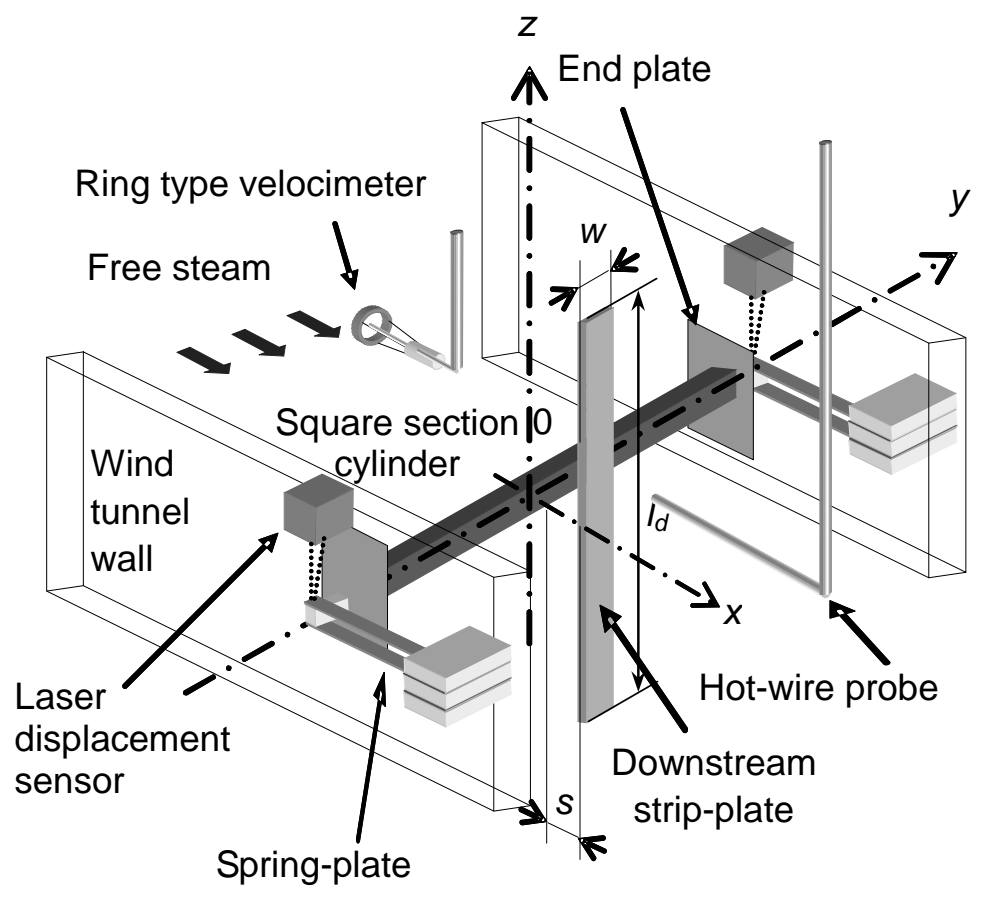

Fig. 1 Arrangement of the experimental apparatus and coordinate system

Table 1 Experimental conditions

\begin{tabular}{lcccccc}
\hline & Fig.2 & $\begin{array}{c}\text { Fig.3, } \\
\text { Fig.4 }\end{array}$ & $\begin{array}{c}\text { Fig.5 } \\
(1 \mathrm{st}, 2 \mathrm{nd})\end{array}$ & Fig.6 & Fig.8 & Fig.9 \\
\hline$d[\mathrm{~mm}]$ & 26 & 26 & 26 & 26 & 26 & 26 \\
& $2,4,6$, & $1,2,3,4$, & 2 & 2 & $1.5,2$ & $1.75,2,3$, \\
$l_{d} / d$ & $10, \infty$ & $6,10, \infty$ & & & & 4,6 \\
$m_{e}[\mathrm{~kg}]$ & 0.15 & 0.15 & 0.15 & 0.15 & 0.15 & 0.15 \\
$k[\mathrm{~N} / \mathrm{m}]$ & 1616 & 1616 & 1616,1618 & - & 1667 & 1667 \\
$\delta$ & 0.03 & 0.03 & 0.03 & - & 0.03 & 0.03 \\
$f_{n}[\mathrm{~Hz}]$ & 16.32 & 16.32 & $16.32,16.37$ & - & 16.03 & 16.03 \\
& & & $1.8-16$, & 7.8 & $2.5-16.5$ & 8.95 \\
\hline$[\mathrm{m} / \mathrm{s}]$ & 3.6 & 7.8 & $2.3-8.4$ & & & -16.93 \\
\hline
\end{tabular}




\section{Nomenclature}

$C_{D} \quad$ Drag coefficient, $=F_{D} /\left\{(1 / 2) \rho U^{2} d L\right\}$

$C_{L} \quad$ Lift coefficient, $=F_{L} /\left\{(1 / 2) \rho U^{2} d L\right\}$

$C_{Z} \quad$ Fluid force coefficient in $Z$-direction, $=F_{Z} /\left\{(1 / 2) \rho U^{2} d L\right\}$

$d \quad$ Side length of square cylinder [mm]

$F_{D} \quad$ Drag force $[\mathrm{N}]$

$F_{L} \quad$ Lift force $[\mathrm{N}]$

$F_{z} \quad$ Fluid force in $Z$-direction [N]

$f_{n} \quad$ Natural frequency for the system $[\mathrm{Hz}]$

$f_{L} \quad$ Frequency of lift force $[\mathrm{Hz}]$

$f_{u} \quad$ Frequency of $x$-component of the flow velocity at the reference position $[\mathrm{Hz}]$

$f_{v} \quad$ Vortex shedding frequency for the elastic system $[\mathrm{Hz}]$

$f_{v 0} \quad$ Vortex shedding frequency for the fixed system $[\mathrm{Hz}]$

$f_{z} \quad$ Oscillation frequency $[\mathrm{Hz}]$

$l_{d} \quad$ Downstream strip-plate length [mm]

$S_{C} \quad$ Scruton number

$S_{L} \quad$ Linear spectrum of lift force $F_{L}$

St $\quad$ Strouhal number

$S_{u} \quad$ Linear spectrum of $x$-component of the flow velocity $u$

$s \quad$ Gap between square cylinder and downstream strip-plate [mm]

$U \quad$ Free flow velocity $[\mathrm{m} / \mathrm{s}]$

$U^{*} \quad$ Reduced flow velocity, $=U / f_{n} d$

$u \quad x$-component of the flow velocity at the reference point in near-wake $[\mathrm{m} / \mathrm{s}]$

$w \quad$ Width of downstream strip-plate [mm]

$w_{c} \quad$ Dissipation energy of damping term during one cycle [J]

$w_{F Z} \quad$ Energy supplied by the exciting force during one cycle [J]

$Z \quad$ Displacement of cylinder in crossflow direction [mm]

$\alpha \quad$ Attack angle of fixed square cylinder [deg.]

$\delta \quad$ Logarithmic damping factor

\section{Subscripts}

rms Root mean square value, representing the amplitude

$0 \quad$ Fixed system

$\infty \quad$ Without downstream strip-plate $(s / d=\infty)$, also denoting $l_{d} / d=\infty$

Time average

\section{Experimental results}

3.1 Influence of downstream strip-plate length on relationship between cylinder vibration and gap $s$

\subsubsection{Influence of short length downstream strip-plate on KVIV}

The downstream strip-plate approached the square cylinder from $s / d=6.0$ at the KVIV velocity $(U=3.6 \mathrm{~m} / \mathrm{s})$. The results are shown in Fig. 2 . The vibration amplitude of the square cylinder $Z_{r m s}$ and the frequency of the $x$-component of the velocity $f_{u}$ are normalized by $Z_{r m s \infty}$ (vibration amplitude without the downstream strip-plate) and the natural frequency $f_{n}$, respectively.

In Region $\mathrm{A}(s / d>4.0)$ in the figure, the vibrations behave almost identically to those of KVIV without the downstream strip-plate, i.e. $f_{u}=f_{n}\left(=f_{v}\right.$ for $\left.\mathrm{KV}\right)$ and $Z_{r m s} \approx Z_{r m s \infty}$. Furthermore, this behaviour is independent of the experimental conditions, showing that the 
downstream strip-plate exerts no effect in this region. In Region $\mathrm{B}(4.0>s / d>2.5-1.5), f_{u}$ and $Z_{r m s}$ continuously and gradually decrease as the strip-plate approaches the cylinder, and $f_{u}$ reduces less when $l_{d} / d$ is smaller. When $s / d$ is smaller than Region $\mathbb{B}$, the behaviours of $f_{u}$ and $Z_{r m s}$ depend on the plate length $l_{d} / d$ as described below.

For the strip-plates of $l_{d} / d=10$ and $l_{d} / d=6, f_{u}$ shows TV shedding frequency in Region D $(2.5>s / d>0.6)$, as also observed when $l_{d} / d=\infty^{(11)}$. However, in Region $\mathrm{E}(s / d<0.4)$, no periodic fluctuations are observed in the velocity $u$. The vibration amplitude $Z_{r m s}$ for the $l_{d} / d=10$ plate is sufficiently suppressed in Regions $\mathrm{D}$ and $\mathrm{E}$. In contrast, $Z_{r m s}$ for the $l_{d} / d=$ 6 plate increases again in Region $\mathrm{D}(2.5>s / d>0.6)$, where it exceeds $Z_{r m s \infty}$, the displacement for $l_{d} / d=\infty$. The maximum amplitude in this region is about 1.4 times that of $l_{d} / d=\infty$, appearing at $s / d \approx 1.4$. As the plate approaches still closer, i.e. enters Region $\mathrm{E}$, the oscillation amplitude reduces to $Z_{r m s} / Z_{r m s \infty}<0.2$, implying that the KVIV is sufficiently suppressed.

For the strip-plates of $l_{d} / d=4$ and $l_{d} / d=2, f_{u}=f_{n}$ in Region $\mathrm{C}(s / d<1.7)$, as observed for KVIV in Region A but different from $l_{d} / d=\infty$ and $l_{d} / d=6$. On the other hand, $Z_{r m s}$ behaves similarly to the case of $l_{d} / d=6$. For the $l_{d} / d=4$ plate, the vibration amplitude increases in Region $\mathrm{C}$ and decreases as the plate moves closer to the cylinder becoming fully suppressed at $s / d<0.8$. For the $l_{d} / d=2$ plate, large-amplitude vibrations occur at smaller $s / d$ values and the vibration suppression region is narrower than for the $l_{d} / d=4$ plate. At $s / d<0.8$, the $l_{d} / d=2$ plate suppresses KVIV to a maximum of $30 \%$ of $Z_{r m s \infty}$ but induces a vibration exceeding $Z_{r m s \infty}$ at $s / d \approx 0.3$.

According to these results, the large-amplitude vibration induced by the $l_{d} / d \leq 6$ plates at $2.0>s / d>1.0$ is similar to the 'Enhanced Karman Vortex Induced Vibration (EKVIV)' observed in circular cylinder/strip-plate systems ${ }^{(9)}$. Vibrations are suppressed less effectively as the strip-plate length decreases. The suppression effect becomes insignificant when $l_{d} / d<2$ plates are used.

\subsubsection{Influence of downstream strip-plate length on galloping and LVIV}

In this experiment, the downstream strip-plate approached the cylinder from $s / d=6.0$ at the galloping velocity $(U=7.8 \mathrm{~m} / \mathrm{s})$. At this velocity, the strip-plate affects the variation of $f_{u}$ with $s / d$ similarly to KVIV in Fig. 2. The results are shown in Fig.3. That is, in Region A $(s / d>4.0)$, the interference effect of the plate is small and $f_{u}$ remains equal to $f_{v}$ of $\mathrm{KV}$, irrespective of $l_{d} / d$. In Region $\mathrm{B}(4.0>s / d>2.5-1.5)$, the KV shedding frequency gradually decreases as the strip-plate approaches the cylinder, the gradient becoming gentler as $l_{d} / d$ is reduced. At $s / d$ smaller than Region $\mathrm{B}$, the behaviour of $f_{u}$ depends on $l_{d} / d$. When $l_{d} / d$ $=10-6, f_{u}$ in Region $\mathrm{D}(1.8>s / d>1.4)$ displays the TV shedding frequency. The TV shedding becomes synchronised with the cylinder oscillation resulting in $f_{u}=f_{n}$ in the TVIV region of $s / d$ with a significant amplitude as indicated in the $Z_{r m s} / Z_{r m s \infty}$ versus $s / d$ plot. For the $l_{d} / d=4-1$ plates, $f_{u}$ displays the KV shedding frequency in Region $\mathbb{C}(s / d<1.8)$.

In contrast, the vibration suppression effect in Fig. 3 is largely different from that of KVIV in Fig. 2, depending on $l_{d} / d$. For the $l_{d} / d=10$ strip-plates, the vibration first decreases at $s / d \approx 6$, becoming fully suppressed at $s / d \leq 5.0$. It should be noted that this suppression effect appears in Region A, where the strip-plate exerts no observable effect on $f_{u}$ and that the original amplitude is reduced to almost zero over a narrow range of $s / d$, i.e. amplitude suppression suddenly occurs at some critical approach distance of the strip-plate. The critical $s / d$ for inducing the suppression effect reduces at shorter plate length $l_{d}$. However, galloping is almost fully suppressed even by the $l_{d} / d=1$ strip-plate, i.e. a square plate equivalent to the cross section of the vibrating cylinder at $2.0>s / d>1.0$.

The TVIV for the $l_{d} / d=10$ strip-plate occurs in almost the same $s / d$ region as $l_{d} / d=\infty$. For the $l_{d} / d=6$ plates, TVIV is observed but its amplitude is much reduced. Plates of $l_{d} / d \leq$ 4 induce no observable TV shedding frequency at any $s / d$ (Fig. 3). 
For the $l_{d} / d=2$ and $l_{d} / d=1$ plates, a large-amplitude vibration, about 3 times and 1.8 times larger than that of a single square cylinder, respectively, occurs at $s / d<1.0$. This vibration appears to be unique to shorter strip-plates and has not been previously reported. Figure 4 shows the spectra of velocity fluctuations $S_{u}$ for the $l_{d} / d=2$ and $l_{d} / d=1$ plates, obtained simultaneously with the results of Fig. 3 . In this figure, the peak frequency $f_{u}$ approximately equals $f_{v}\left(\gg f_{n}\right)$ at $s / d \geq 1.0$ for both plates. At $s / d<1.0$, the spectra of both plates display peaks at $f_{v}$ of the fixed system and around $f_{n}$. However, while the frequency $f_{u}$ of the latter peak for the $l_{d} / d=2$ plate is almost equal to $f_{n}$, that of the $l_{d} / d=1$ is considerably deviating from $f_{n}$. These results show that the large-amplitude vibrations for the $l_{d} / d=1,2$ plates in Region $\mathrm{E}$ of Fig. 3 are not induced by vortices. Hence in this paper, this vibration is called 'Wake Body Interference Vibration (WBIV)' and will be discussed in Section 3.2 .

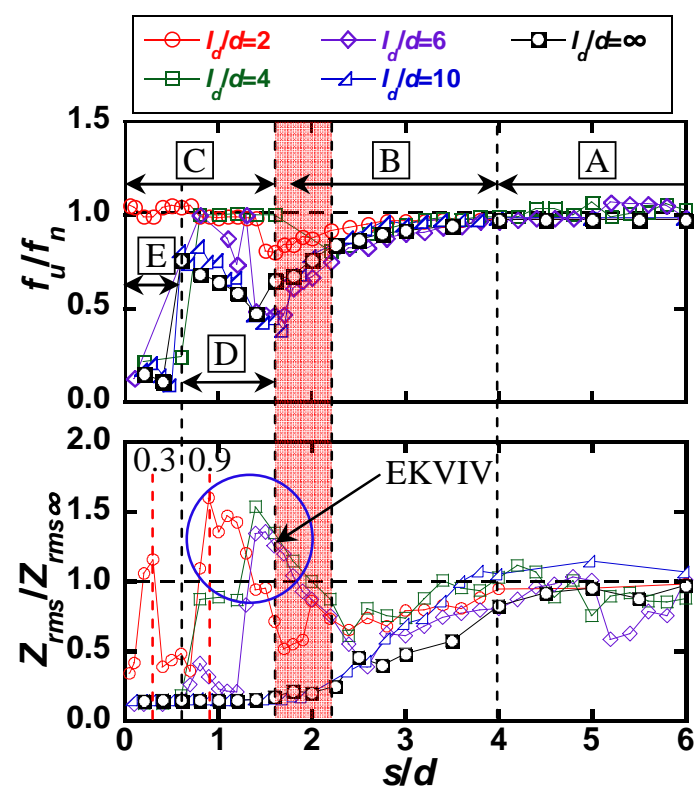

Fig. 2 Influence of downstream plate length $l_{d}$ on $\operatorname{KVIV}(U=3.6 \mathrm{~m} / \mathrm{s})$

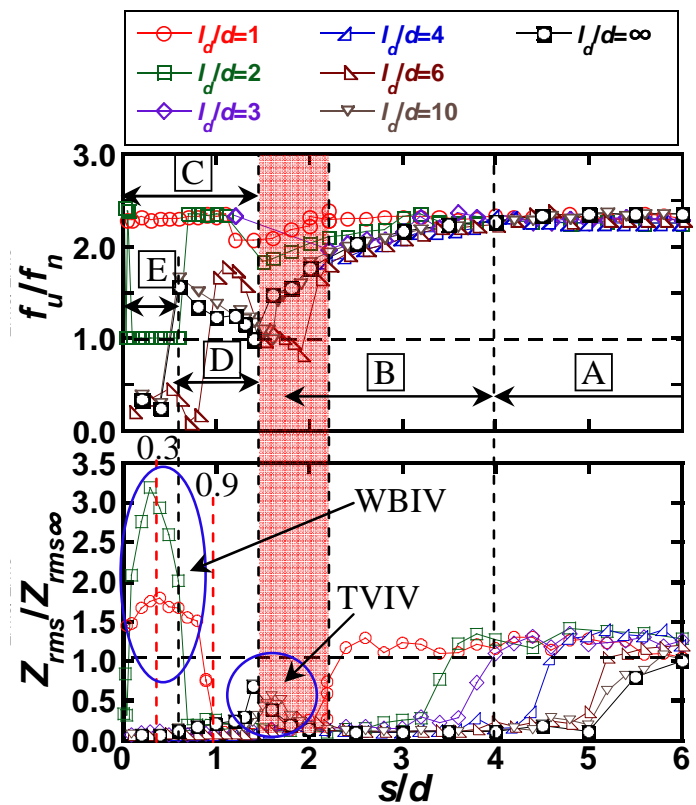

Fig. 3 Influence of downstream plate length $l_{d}$ on galloping $(U=7.8 \mathrm{~m} / \mathrm{s})$ 

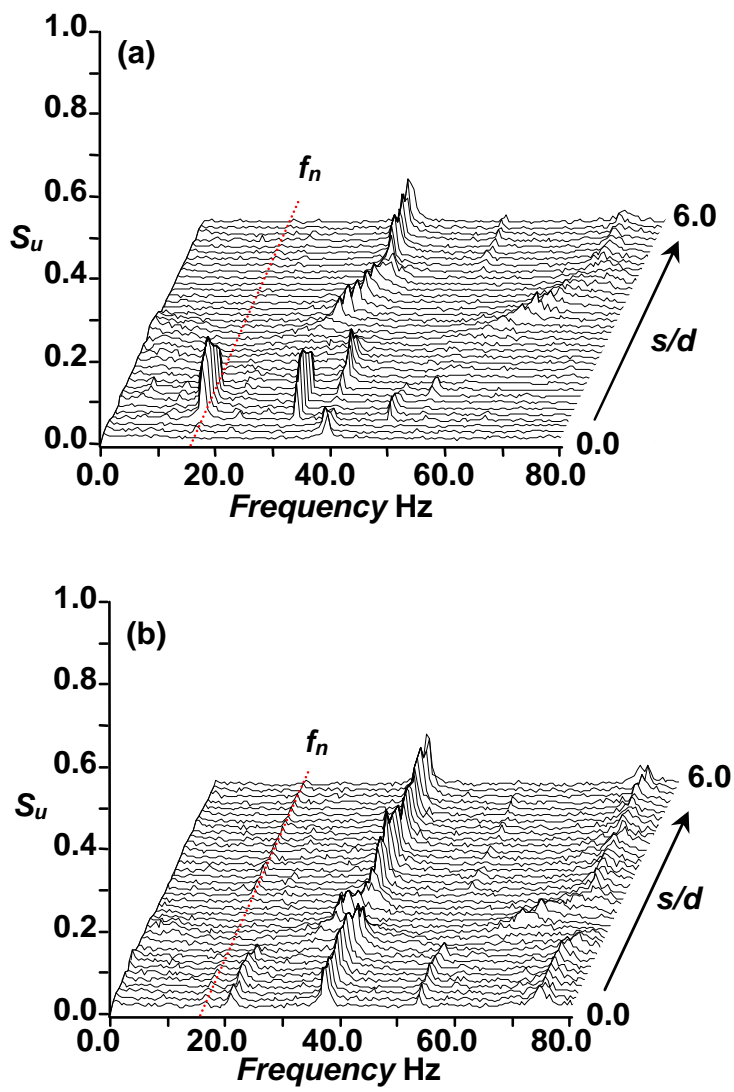

Fig. 4 Influence of downstream strip-plate on $S_{u}$ (a) $l_{d} / d=2$, (b) $l_{d} / d=1(U=7.8 \mathrm{~m} / \mathrm{s})$

\subsection{Examination of Wake Body Interference Vibration induced by short strip-plate}

\subsubsection{Vibration behaviour with varying flow velocity}

As described in the preceding sections, the $l_{d} / d=2$ strip-plate placed in the wake of cylinder exerts an influence on vibrations that depends on $s / d$. Hence, the fluctuation velocity $u$ and the displacement $Z$ of the square cylinder were measured for varying flow velocity $U$ with $s / d$ fixed at $0.9,0.3$ and 2.0. The selected $s / d$ are representative of the gaps that induce large-amplitude KVIV (EKVIV) in Fig. 2, large-amplitude WBIV at the galloping velocity in Fig. 3 and full suppression of galloping, respectively. The results are plotted in Fig. 5, where the experiment was repeated at $s / d=0.9$ and 0.3 and are compared with those of the single square cylinder.

At $s / d=0.9, f_{u}\left(=f_{v}\right.$ of $\left.\mathrm{KV}\right)$ equals $f_{n}$ in the velocity range $(3.4 \leq U \leq 4.0-4.4) \mathrm{m} / \mathrm{s}$ accompanied by KVIV, as observed for the single square cylinder but occurring at much higher amplitude. At higher flow velocities $(U=5.3 \mathrm{~m} / \mathrm{s}-6.0 \mathrm{~m} / \mathrm{s})$, another large vibration appears, whose amplitude differs between the two measurements. The amplitude of this vibration rapidly increases with flow velocity and suddenly declines at around $U=7.0 \mathrm{~m} / \mathrm{s}$, becoming constant at $U>9.0 \mathrm{~m} / \mathrm{s}$. Within both large-amplitude ranges of flow velocity, the dominant frequency $f_{u}$ in the velocity spectrum is almost constant at $f_{u}=f_{n}$. As the amplitude becomes small at $U>9.0 \mathrm{~m} / \mathrm{s}, f_{u}$ approximates the $\mathrm{KV}$ shedding frequency in the fixed system $f_{v 0}$. These results indicate that the second large vibration in the higher velocity range is not induced by $\mathrm{KV}$, since the natural vortex shedding frequency $f_{v 0}$ far exceeds the vibration frequency. At present, the cause of this vibration is unclear and is left for future investigations.

At $s / d=0.3, f_{u}$ and $f_{n}$ are equal and the relationship between $Z_{r m s}$ and $U$ is similar to that 
of the single square cylinder in the velocity range $(3.4 \leq U \leq 4.0) \mathrm{m} / \mathrm{s}$. Hence, the large-amplitude vibration observed for $l_{d} / d=2$ at $s / d=0.3$ in Fig. 2 is considered as KVIV. When $U \leq 5.0 \mathrm{~m} / \mathrm{s}$, the vibration amplitude is small and $f_{u}$ equals $f_{v 0}$ except in the KVIV regions. At $U>5.0 \mathrm{~m} / \mathrm{s}-5.6 \mathrm{~m} / \mathrm{s}$, a new vibration occurs whose amplitude divergently increases with increasing flow velocity. This vibration is first incited at significantly lower velocity than the galloping velocity of a single square cylinder. Throughout its duration, the dominant frequency $f_{u}$ in the velocity $u$ is $f_{n}$.

At $s / d=2.0$, KVIV occurs in the velocity range $(3.4 \leq U \leq 4.2) \mathrm{m} / \mathrm{s}$ but at much smaller amplitude than at $s / d=0.3$ or 0.9 . In the velocity range $(4.2<U<10.3) \mathrm{m} / \mathrm{s}$, no vibration is observed and $f_{u}$ is slightly smaller than $f_{v 0}$. Velocities around $U=10.3 \mathrm{~m} / \mathrm{s}$ incite a weak vibration whose amplitude rises as $U$ further increases, eventually becoming comparable with the $s / d=0.9$ regime at $(14.9<U<15.6) \mathrm{m} / \mathrm{s}$. At still higher velocities, amplitude of the vibration decreases. These results show that both KVIV and galloping can be suppressed by specifying $s / d=2.0$.

Thus, the results in Fig. 5 indicate that vibrations in the KVIV velocity range but with an amplitude larger than KVIV induced by the $l_{d} / d=2$ plate at $s / d=0.9$ can be regarded as enhanced KVIV (EKVIV). The large-amplitude vibration in the range of galloping flow velocity at $s / d=0.3$ behaves like a fluid elastic vibration (FEV). However, at the present stage we cannot ascertain whether this vibration is VIV or galloping.

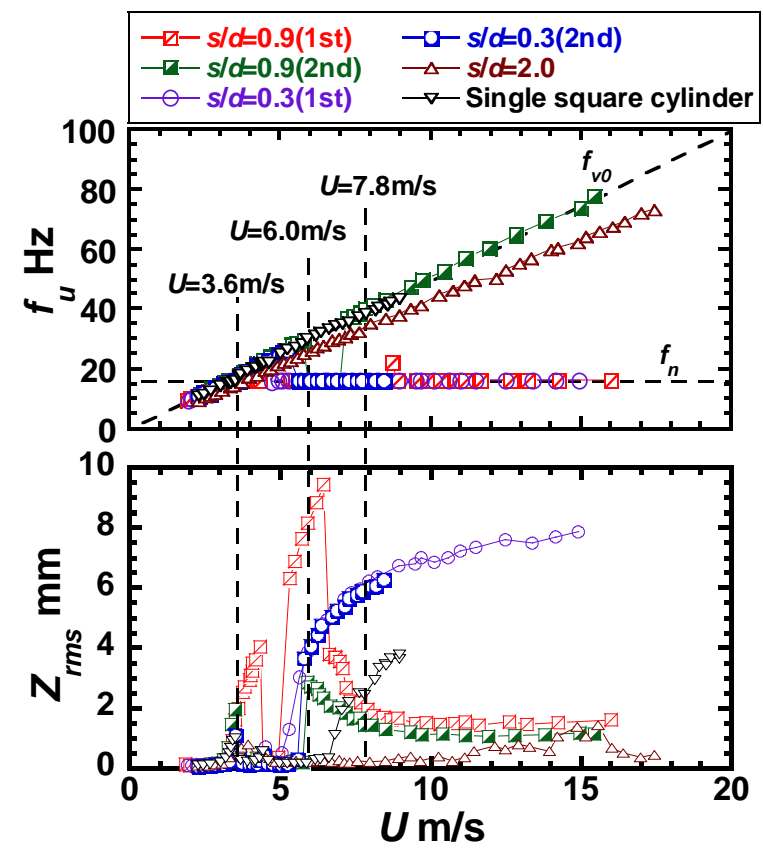

Fig. 5 Variation of $Z_{r m s}$ and $f_{u}$ with $U$ at $s / d=0.3,0.9$ and 2.0 for the $l_{d} / d=2$ plate.

$1^{\text {st }}$ and $2^{\text {nd }}$ denote that two experiments were conducted under the same conditions.

\subsubsection{Fluctuating lift force on fixed square cylinder}

To examine whether WBIV induced by the $l_{d} / d=2$ plate at $s / d=0.3$ in Fig. 3 arises from vortices, the lift force $F_{L}$ and the fluctuating flow velocity $u$ were measured in the fixed system. In Fig. 6, the linear spectra of $u$ and $F_{L}$, designated $S_{u}$ and $S_{L}$ respectively, are shown for various $s / d$ as the $l_{d} / d=2$ strip-plate approaches from $s / d=6.0$ at $U=7.8 \mathrm{~m} / \mathrm{s}$.

At all $s / d, S_{u}$ develops an obvious peak at a certain frequency $f_{u}$ and a dull peak at $2 f_{u}$ (see Fig. 6(a)). By contrast, $S_{L}$ shows a single sharp peak at $f_{L}=f_{u}$ (Fig. 6(b)). The Strouhal numbers $S t_{u}$ and $S t_{L}$, defining the non-dimensional frequencies of $u$ and $F_{L}$ respectively, at $s / d>2.7$ and $s / d<1.6$, approximate the Strouhal number of the Karman vortex shedding frequency $S t_{0}=0.13$ for a single fixed square cylinder. Hence, it is re-confirmed that $f_{u}$ in 
Regions A, B and C of Figs. 2 and 3 is the Karman vortex frequency. By contrast, no peak around $f_{n}$ appears in Fig. 6 in $s / d$ regions corresponding to Region $\mathrm{E}$ in Figs. 3 and 4. These results verify that the sharp peaks at $f_{u}=f_{n}$ occurring at $s / d=0.1-0.6$ in Fig. 4 (a) for the elastically supported square cylinder are caused by the oscillation of the square cylinder and not by Karman vortex shedding. Therefore, the vibration observed at $s / d<1.0$ in Fig. 3, i.e. WBIV is attributable to fluid-elastic instability, but not to periodic vortex shedding.
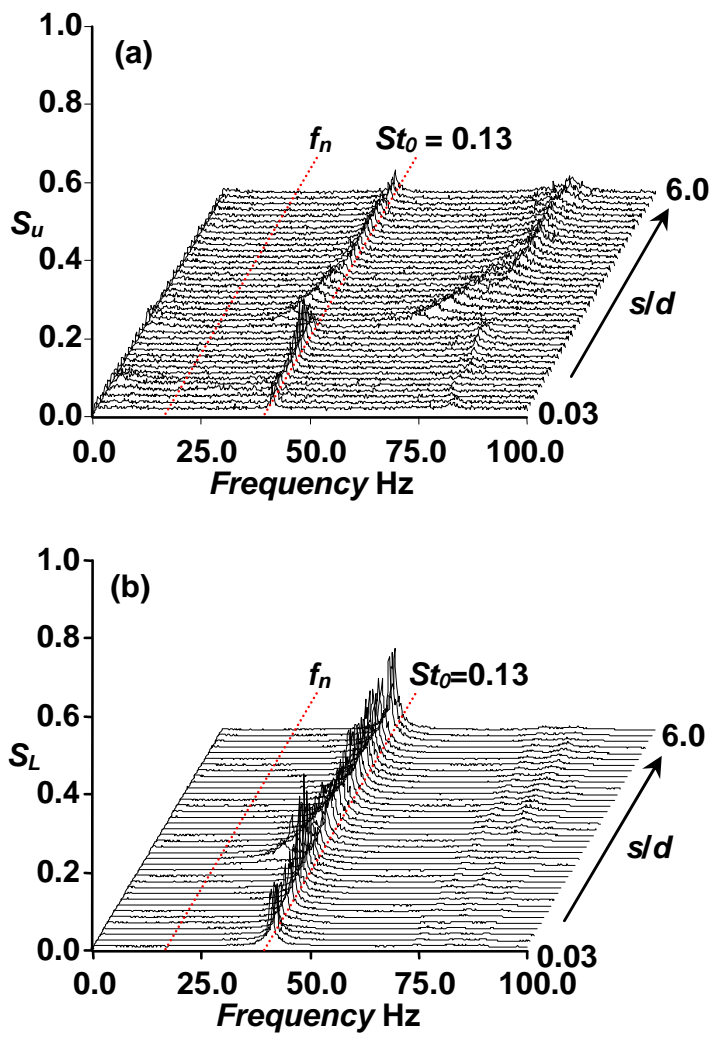

Fig. 6 Spectra of $u$ and $F_{L}$ in a fixed system with $l_{d} / d=2$ strip-plate $(U=7.8 \mathrm{~m} / \mathrm{s})$ :

(a) $S_{u}$ and (b) $S_{L}$

\subsection{Verification of WBIV as a Fluid-Elastic-Instability vibration}

\subsubsection{Effect of position of downstream plate relative to the cylinder}

Fluid-elastic-instability vibrations are induced by instability of the system to the displacement or the velocity of the body motion. Therefore, the WBIV shown in Section 3.2 may be incited by instability to the location of the downstream plate relative to the square cylinder, i.e. the relative displacement of the plate may generate a fluid force that enhances cylinder motion. To test this idea, a plate was affixed to the square cylinder with a bracket in a T-shaped fin, as shown in Fig. 7. The effect of this plate on galloping was investigated considering the bracket length and gap $s$ to be equivalent. The bracket and the plate were fabricated from planar balsa wood of thickness $5 \mathrm{~mm}$ and width $w=d$.

This test was initially conducted on two plates of length $l_{d} / d=2$ and 1.5 ; the dimensions at which WBIV is most remarkably observed. The velocity $u$ and displacement $Z$ were measured at a galloping flow velocity $U=8.95 \mathrm{~m} / \mathrm{s}$ using T-fins at $s / d=0.2-6.0$. A preliminary experiment conducted on the brackets alone showed that the brackets exert negligible effect on vortex shedding and cylinder vibration over a range of flow velocities. The vibration amplitude normalized by the amplitude at $s / d=6.0$ is plotted against $s / d$ in Fig. 8. The results are compared with those obtained for the detached plate, i.e. the square-cylinder/strip-plate system, indicated as 'D-plate'. For the T-fin of $l_{d} / d=1.5$, the 
vibrations behave almost identically to those of the detached counterpart, i.e. galloping is suppressed at $1.4<s / d<2.0$ and vibrations are amplified at $s / d<1.4$. The maximum amplitude at $s / d=0.3$ is 1.5 times that of the detached counterpart. When the dimensions of the T-fin are altered to $l_{d} / d=2.0$, the galloping suppression is comparable to that of the detached counterpart but no vibration is induced at $s / d<1.4$.

Based on the above result, T-fins of $s / d=0.3$ and lengths $l_{d} / d=1-6$ were tested at the galloping velocity $U=8.94 \mathrm{~m} / \mathrm{s}$. The results (Fig. 9), are compared to those of the detached counterpart. To compensate for differences between the experimental conditions (Table 1), the non-dimensional amplitude was multiplied by the Scruton number Sc. In this figure, almost no vibration is discernible at $l_{d} / d \geq 3$ for either the T-fin or the detached plate, confirming that the galloping suppression effect is equivalent between the two cases. However, agreement among the WBIV induced by the shorter plates is qualitative rather than quantitative, since the maximum vibration appears at $l_{d} / d=1.5$ for the T-fin but at $l_{d} / d$ $=2.0$ in the detached plate system.

The results presented in this section exclude the relative position in the strip-plate/square-cylinder system as an essential cause of WBIV.

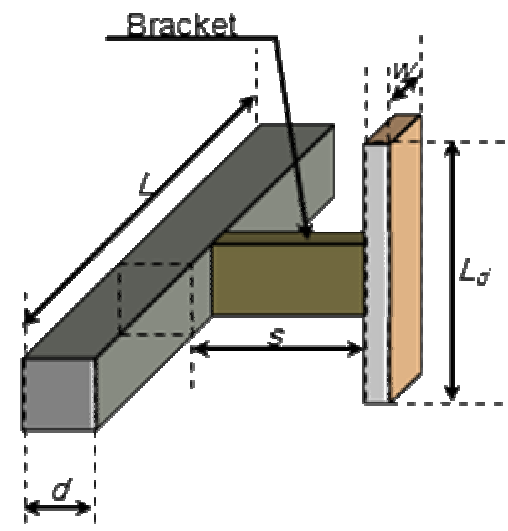

Fig. 7 Arrangement of the T-shaped fin

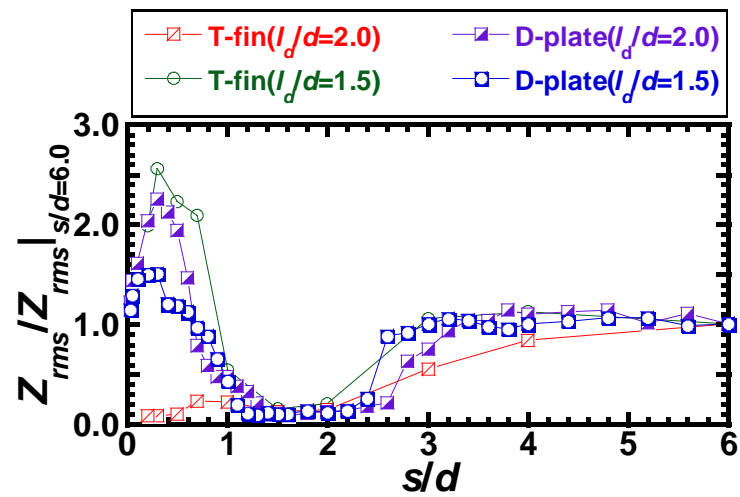

Fig. 8 Effect of the T-shaped fin on galloping $(U=8.95 \mathrm{~m} / \mathrm{s})$ 


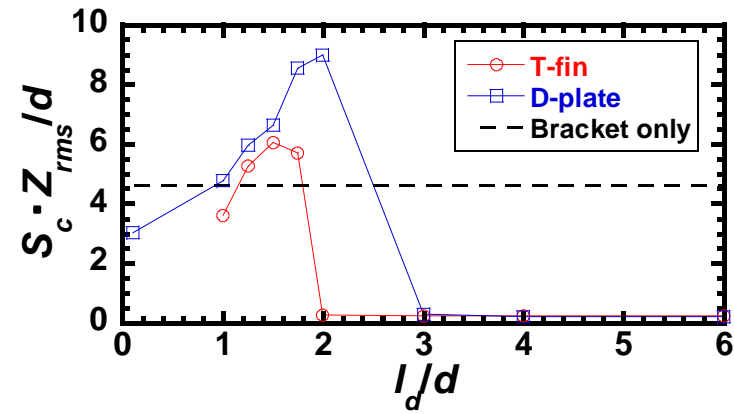

Fig. 9 Relationship between $l_{d} / d$ and WBIV $(s / d=0.3, U=8.94 \mathrm{~m} / \mathrm{s})$

\subsubsection{Prediction of WBIV induced by a short plate}

FIVs are generally difficult to predict since the fluid force $F_{z}(t)$ exerted on a vibrating body cannot be estimated. However, the galloping, a typical vibration in FEV category, can be predicted from the quasi-steady approximation, in which the exciting force on the vibrating body is assumed equivalent to the time-averaged fluid force acting on the same stationary body at an attack angle given by Eq. (1). Applying this approximation, the exciting force $\overline{F_{Z}}$ is the $z$-component of the vector sum of the time-averaged lift $\overline{F_{L}}$ and the drag $\overline{F_{D}}$.

$$
\alpha=\tan ^{-1}(-\dot{Z} / U)
$$

In this method, $\overline{F_{L}}$ and $\overline{F_{D}}$ are obtained either computationally or experimentally. The subsequently obtained $\bar{F}_{Z}$ is approximated by a polynomial of $3^{\text {rd }}$ to $7^{\text {th }}$ order. Applying this $\overline{F_{Z}}$ to the equation of motion of the body, the vibration amplitude is analytically or numerically obtained.

However, since the above approximation for $\overline{F_{Z}}$ is not generalisable, here we propose a new method that predicts WBIV from energy considerations.

When a square cylinder oscillates with period $T$, the work done by the exciting force $F_{z}$ during a single cycle is given by

$$
w_{F z}=\int_{0}^{T} F_{Z} \dot{Z} d t
$$

The energy dissipated during a cycle by a damper with damping factor $C_{e}$ is

$$
w_{c}=C_{e} \int_{0}^{T} \dot{Z}^{2} d t
$$

If the oscillation is steady, the oscillation amplitude $Z_{A}$ should satisfy the following condition.

$$
w_{F z}=w_{c}
$$

If the oscillation is given by

$$
Z=Z_{A} \sin \left(2 \pi f_{n} t\right)
$$

the energy dissipated by the damper is proportional to the square of $Z_{A}$ and is written as

$$
w_{c}=2 \pi^{2} C_{e} Z_{A}^{2} f_{n}
$$


Now, the quasi-steady approximation for galloping is assumed to also hold for WBIV. Under this condition, $w_{F Z}$ can be expressed as a function of $Z_{A}$. The exciting force $\overline{F_{Z}}$ at some phase $\theta=2 \pi f_{n} t$ during the oscillation cycle is then estimated through the following procedure.

1) Specify $Z_{A}$ and calculate $Z$ and $\dot{Z}$ at phase value $\theta$.

2) Obtain $\alpha$ from Eq. (1).

3) Obtain $\overline{F_{L}}(Z, \alpha)$ and $\overline{F_{D}}(Z, \alpha)$ from wind tunnel experiments and calculate $\overline{F_{Z}}(Z, \alpha)$ as the $\mathrm{z}$-component of their resultant.

4) Iterate steps 2)-3) from $\theta=0$ to $2 \pi$.

5) Iterate steps 1)-4) over an appropriate expected region of $Z_{A}$.

Strict implementation of the above procedure requires a prohibitive number of wind tunnel experiments. In this work, based on the T-fin results presented in Section 3.3.1, $\overline{F_{Z}}(Z, \alpha)$ is assumed independent of $Z$. Adopting this simplification, the experiments are conducted at an appropriately selected $Z_{A}$ and the results are inserted into Eq. (7) to obtain the exciting force coefficient. $\overline{C_{Z}}$ thus obtained is considered applicable to arbitrary $Z_{A}$.

$$
\overline{C_{Z}}=\overline{C_{L}} \cos \alpha+\overline{C_{D}} \sin \alpha
$$

In this work, $\overline{F_{L}}$ and $\overline{F_{D}}$ were measured in wind tunnel experiments at $Z_{A}=7 \mathrm{~mm}$ decided from Fig. 5. The exciting force coefficient $\overline{C_{Z}}$ calculated from these results is plotted against $\theta=2 \pi f_{n} t$ in Fig. 10. In this figure, the fluid force $\overline{F_{Z}}$ acts in the direction of body motion in the red-hatched region ' $\mathrm{A}$ ', supplying energy to the body to sustain steady oscillations. The result for $\overline{C_{z}}$ is approximated by the solid curve, which is used to calculate $Z_{A}$ at various $U$ through the above described procedure. The result is compared with measurement in Fig. 11 in which the amplitude $Z_{A}$ is modified as $Z_{A}=\sqrt{ } 2 Z_{r m s}$. The predicted and measured results favourably agree in the velocity region $(5 \leq U \leq 10.7) \mathrm{m} / \mathrm{s}$. At higher velocities, the predicted $Z_{A}>\left(l_{d}-d\right) / d$ indicating that the square cylinder moves beyond the region of $z$ covered by the downstream plate at the peak of the oscillation. It is reasonable to consider that the influence of $Z$ on $F_{z}$ is non-negligible at such velocities causing the deviation of the prediction from measurement. Because the observed agreement between the prediction and measurement verifies that WBIV is induced by the fluid elastic instability, this vibration may reasonably be termed 'Wake Body Interference Fluid Elastic Vibration (WBIFEV)'. 


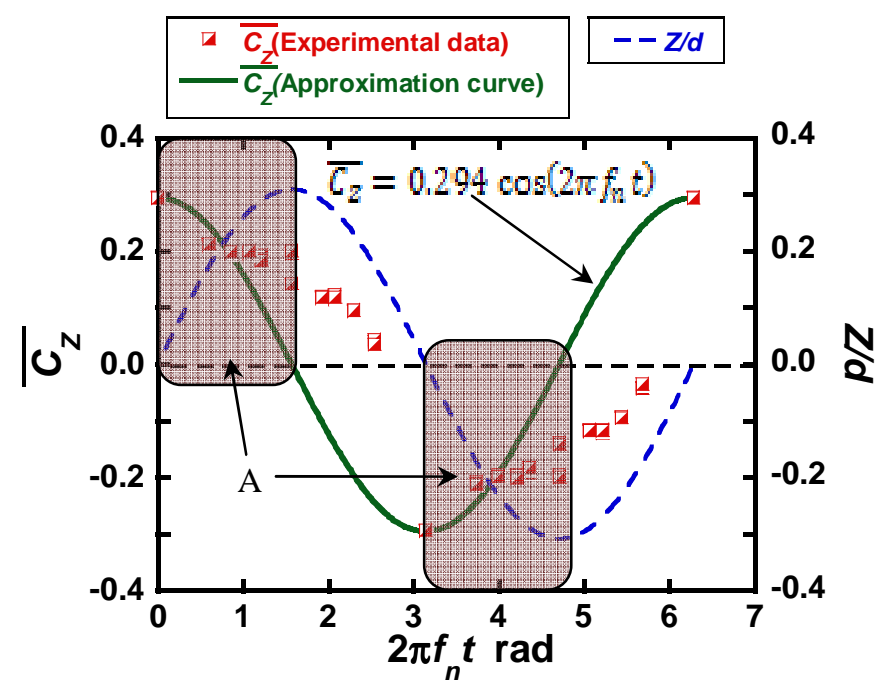

Fig. 10 Fluid force coefficient and displacement as functions of phase $2 \pi f_{n} t$

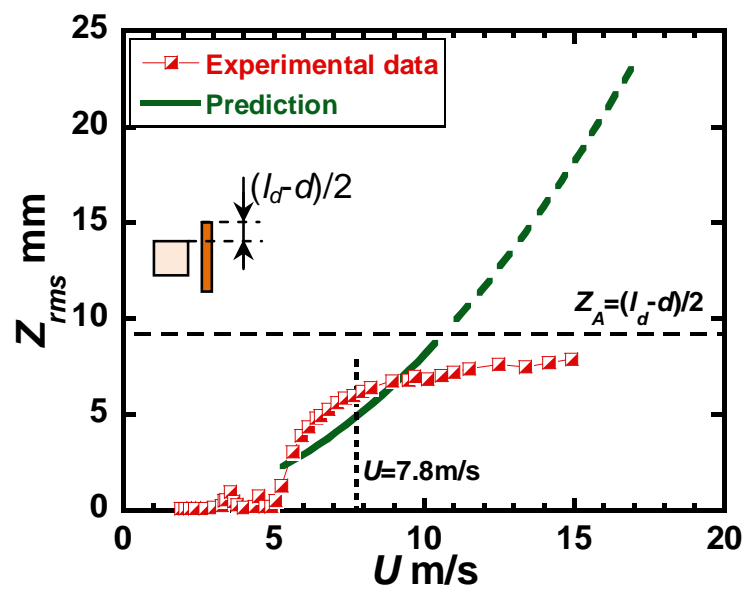

Fig. 11 Comparison between WBIFEV predictions and the results of wind tunnel experiments $\left(l_{d} / d=2, s / d=0.3\right)$

\subsection{Classifying the influences of strip-plate on vibration mapped on the $\boldsymbol{l}_{\boldsymbol{d}} / \boldsymbol{d}-\mathrm{s} / \boldsymbol{d}$ plane}

Figures 12 and 13 show the various influences of the strip-plate on KVIV and galloping described in the preceding sections, by presenting them as domains in the $l_{d} / d-s / d$ plane. In these figures, $[s / d]_{0.2}$ and $[s / d]_{0.8}$ denote the $s / d$ at which the amplitudes $Z_{r m s}$ are 0.2 and 0.8 times that of the isolated counterpart $Z_{r m s \infty}$, respectively. In this context, $[s / d]_{0.8}$ is a measure of the non-dimensional gap at which KVIV or galloping is first suppressed as the strip-plate approaches from infinitely far downstream and $[s / d]_{0.2}$ represents the maximum $s / d$ at which vibrations are sufficiently suppressed. The domain of EKVIV is defined as the region where $Z_{r m s}>Z_{r m s \infty}$ while that of WBIFEV is the region of $Z_{r m s}>0.2 Z_{r m s \infty}$ at $s / d \leq 1$.

According to Fig.12, the non-dimensional gap $[s / d]_{0.8}$ at which KVIV suppression is initiated depends only slightly on the plate length. For instance, the $[s / d]_{0.8}$ of the $l_{d} / d=\infty$ and $l_{d} / d=2$ plates ( $\approx 3.8$ and 3 , respectively) differs by 0.8 . On the other hand, the non-dimensional gap for sufficient KVIV suppression, $[s / d]_{0.2}$, is significantly reduced for shorter plates, and vanishes at $l_{d} / d<4$. EKVIV occurs at $s / d=0.8-2.6$ and $l_{d} / d=2-6$ (red-hatched area in Fig. 12). The domain of $s / d$ over which $Z_{r m s}>Z_{r m s \infty}$ shifts to smaller values as the plate length reduces. 
At galloping velocity (Fig.13), the $[s / d]_{0.8}$ and $[s / d]_{0.2}$ that characterise the vibration suppression effect also decrease as plate length decreases. However, in this case the shortest plate $l_{d} / d=1$ can sufficiently suppress the vibration and the differences between $[s / d]_{0.8}$ and $[s / d]_{0.2}$ are considerably smaller than those of Fig.12, revealing that galloping can be suppressed by a slight gap reduction. At the galloping velocity, other than KVIV and galloping, two new vibration phenomena emerge; one is the longitudinal vortex induced vibration (LVIV) caused by the downstream strip-plate at $l_{d} / d \geq$ and $s / d=1.0-1.7$, and the other is WBIFEV appearing at $l_{d} / d=1-2$ and $s / d<1$.

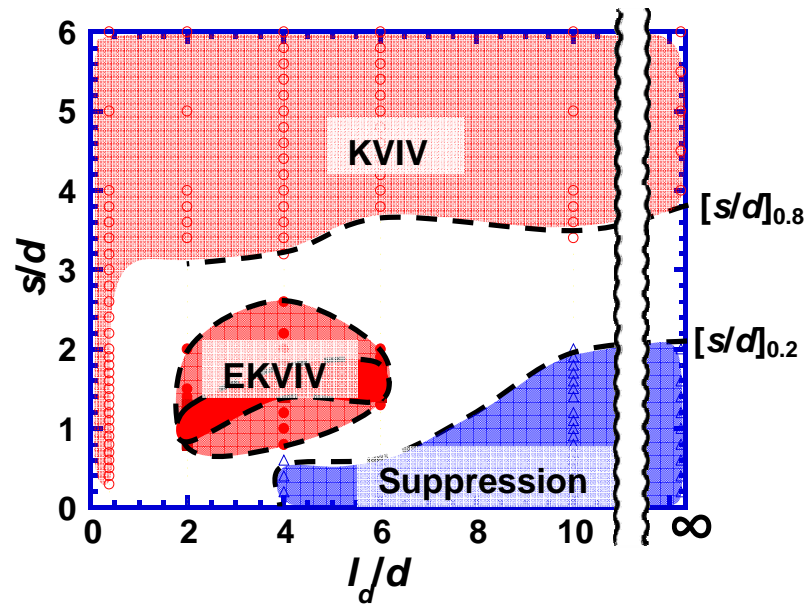

Fig. 12 Vibration map of square cylinder on $l_{d} / d-s / d$ plane under KVIV $(w / d=1.0, R e=6200)$. The bright red region is the domain of $s / d$ for which $Z_{r m s}>Z_{r m s \infty}$

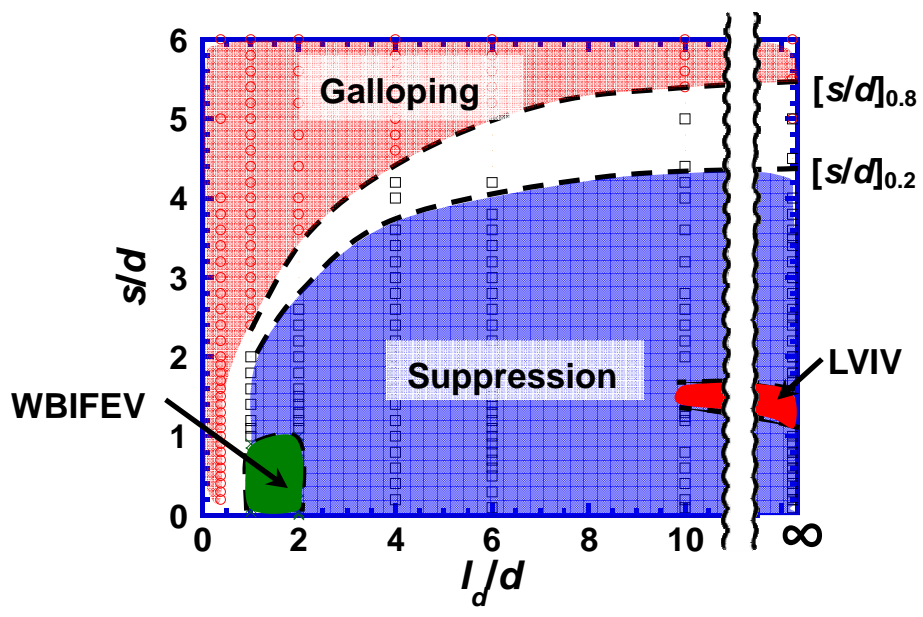

Fig. 13 Vibration map of square cylinder on $l_{d} / d-$ s/d plane under galloping $(w / d=1.0, R e=13400)$. The red and green regions delineate new vibrational phenomena that emerge under galloping conditions

\section{Concluding remarks}

In our previous report, the crossflow vibration of a square cylinder of side length $d$ was found to be largely affected by a cruciform arrangement of a downstream strip-plate of width $w=d$ and virtually infinite length. In this work, a finite-length strip-plate was placed downstream of the cylinder and its effect on the cylinder vibration was investigated through wind tunnel experiments. The aim was to achieve vibration control.

The results are summarized as follows 
1) The KVIV is less suppressed by shorter strip-plates and becomes insignificant when $l_{d} / d<2$. On the contrary, KVIV is enhanced by strip-plates of $l_{d} / d<6$ within a range of non-dimensional gap sizes $s / d$. This vibration has been termed Enhanced KVIV (EKVIV).

2) Galloping of square cylinder is effectively suppressed by short strip-plates. Even a square strip-plate $\left(l_{d} / d=1\right)$ almost fully suppresses galloping at $s / d 1.0-2.0$.

3) The trailing-vortex induced vibration (TVIV) reduces as the strip-plate is made shorter and is absent for strip-plates of $l_{d} / d<4$.

4) A large-amplitude vibration is induced when strip- plates of $l_{d} / d \leq 2$ are located at $s / d \leq$ 1.0. Because this newly found vibration appears to be caused by fluid-elastic instability, we have named it 'Wake body interference fluid elastic vibration (WBIFEV)'.

These phenomena are expected to depend on the aspect ratio of the square cylinder and the span-wise location of the downstream strip-plate. The WBIFEV presents a challenging problem to fluid mechanics, since it appears to be a new type of FEV different from galloping. This vibration is important from a practical engineering perspective since it may be hazardous, leading to accidents, or beneficial, to be exploited in energy harvesting. Hence, clarifying how downstream bodies affect the vibrations of upstream cylinders is an important goal of future research.

\section{References}

(1)G.V. Papaioannou, D.K.P. Yue, M.S. Triantafyllou, and G.E. Karniadakis, On the effect of spacing on the vortex-induced vibrations of two tandem cylinders, Journal of Fluids and Structures, Vol.24, Issue 6 (2008), pp. 833-854.

(2)Sangil Kim, Md. Mahbub Alam, Hiroshi Sakamoto, and Yu Zhou, Flow-induced vibration of two circular cylinders in tandem arrangement. Part 1: Characteristics of vibration, Journal of Wind Engineering and Industrial Aerodynamics, Vol.97, Issue 5-6 (2009), pp. 304-311.

(3)Sangil Kim, Md. Mahbub Alam, Hiroshi Sakamoto, and Yu Zhou, Flow-induced vibration of two circular cylinders in tandem arrangement. Part 2: Suppression of vibrations, Journal of Wind Engineering and Industrial Aerodynamics, Vol.97, Issue 5-6 (2009), pp. 312-319.

(4)I. Korkischko, and J.R. Meneghin, Experimental investigation of flow-induced vibration on isolated and tandem circular cylinders fitted with strakes, Journal of Fluids and Structures, Vol.26, Issue 4 (2010), pp. 611-625.

(5)B.H.L. Gowda, and R. Ajith Kumar, Flow-induced oscillations of a square cylinder due to interference effects, Journal of Sound and Vibration, Vol.297, Issues 3-5, (2006), pp. 842-864.

(6)R. Galvao, E. Lee, D. Farrell, F. Hover, M. Triantafyllou, N. Kitney, and P. Beynet, Flow control in flow-structure interaction, Journal of Fluids and Structures, Vol.24, Issue 8 (2008), pp. 1216-1226.

(7)M. Shirakashi, K. Mizuguchi, and H.M. Bae, Flow-induced excitation of an elastically-supported cylinder caused by another located downstream in cruciform arrangement, Journal of Fluids and Structures, Vol.3, Issue 6 (1989), pp. 595-607.

(8)M. Shirakashi, T. Takahashi, I. Kumagai, and T. Matsumoto, Vortex-induced vibration of the upstream cylinder of a two-cylinder system in cruciform arrangement, Journal of Computational and Applied Mechanics, Vol.2, No.1 (2001), pp.103-122.

(9) M. Koide, N. Kato, T. Takahashi, and M. Shirakashi, Vibration Control for a Circular Cylinder by a Strip-Plate Set Downstream in Cruciform Arrangement: 2nd Report, Generation and Suppression of Vortex Excitation on Elastically Supported Cylinder (Fluids Engineering), Japan Society of Mechanical Engineering Series. B, Vol.75, No.752, (2009), pp. 691-669 (in Japanese).

(10)M. Koide, N. Kato, S. Yamada, Y. Kawabata, T. Takahashi, and M. Shirakashi, Influence of a 
cruciform arrangement downstream strip plate on crossflow vibration of a square cylinder, Journal of Computational and Applied mechanics, Vol.8, No.2 (2007), pp.135-148.

(11)Y. Kawabata, T. Takahashi, and M. Shirakashi, Influence of a Downstream Strip-Plate on Galloping of a Square Cylinder in Cruciform Arrangement (Fluids Engineering), Japan Society of Mechanical Engineering Series. B, Vol.75, No.754 (2009), pp. 1304-1309 (in Japanese).

(12)M. Koide, T. Takahashi, and M. Shirakashi, Development of a Ring-Type Vortex Anemometer for Low-Velocity Wind Tunnel Experiments, Japan Society of Mechanical Engineering Series. B, Vol.67, No.657 (2001), pp. 1105-1111 (in Japanese).

(13)R.D. Blevins, Flow-Induced Vibration (second edition), (1990), pp.104-120., Van Nostrand reinhold. 